\title{
Stimulus stringing by pigeons: Effect of feedback for correct selections
}

\author{
W. KIRK RICHARDSON and B. J. BITTNER \\ Georgia State University, Atlanta, Georgia 30303
}

\begin{abstract}
Previous studies have shown that pigeons could learn a serial list of colors and to select each color in the proper order when presented with an array of colors. Errors of jumping forward in the required sequence (forward errors) were most probable, with jumps backward in the required sequence (backward errors) being relatively improbable. One proposed explanation for the higher probability of forward errors suggests that the pigeon pecks at the correct stimulus without activating the response switch and then pecks the next stimulus in the sequence closing the response switch. This "inadequate-peck" hypothesis suggests that forward errors are due to weak stimulus control and that increased feedback for correct pecks should therefore reduce forward error probability relative to a low-feedback condition. The present study compared responding under conditions of high and no experimenter-provided feedback for correct pecks. As the feedback did not affect the probability of forward errors, the inadequate-peck hypothesis was not supported. A short-term memory explanation is consistent with the data.
\end{abstract}

Two recent studies have shown that pigeons can learn to select colors in an experimenter-defined order when the array of colors remains displayed throughout the trial (Richardson \& Warzak, 1981; Straub, Seidenberg, Bever, \& Terrace, 1979). In the Richardson and Warzak study, the number of colors to be ordered was increased by adding one color at a time until the string was four colors long. At the beginning of each trial, the four colors were presented in randomly selected locations on a row of five keys. Pecking each color once, in the correct order, ended the trial, and another trial was given after an intertrial interval. Each correct response resulted in two experimenter-controlled sources of feedback which remained present throughout the trial: namely, the correctly selected color increased in brightness and the same color was presented in the leftmost unoccupied position of a row of five stimulus display windows located just above the keys. A peck to the unlighted key or to any color other than the one defined as correct at that point in the sequence resulted in brief auditory feedback and termination of the trial. After a brief intertrial interval, the same array was presented again (a correction procedure), with the bird required to start at the beginning of the string.

Although there were several differences in the details of the Richardson and Warzak and the Straub et al. studies, both found that the most probable error

This study was based on a thesis submitted by the second author to the Department of Psychology, Georgia State University, in partial fulfillment of the requirements for the MA degree. We wish to thank the staff of the Georgia State University Computer Center for technical assistance. Reprints may be obtained from W. Kirk Richardson, Psychology Department, University Plaza, Georgia State University, Atlanta, Georgia 30303. was a jump forward in the required sequence (e.g., selecting the fourth stimulus when the second stimulus was correct) and that a jump backwards in the sequence (e.g., selecting the first stimulus when the third stimulus was correct) was relatively improbable. The Richardson and Warzak study would allow an interpretation of the relatively high probability of forward errors as being the direct result of a discrimination based on lasting feedback for correct selections. A bright/dim discrimination could develop since only dim stimuli were correct; selection of any bright stimulus resulted in a brief auditory feedback, termination of the trial, and never any food. Such a bright/dim discrimination could be responsible for the low probability of backward errors.

In the Straub et al. (1979) study, subjects were trained with feedback for correct selections, but the feedback was dropped prior to the final test condition. No data comparing the feedback and no-feedback conditions were presented. However, a comparison of their results with those of Richardson and Warzak (1981) showed that both studies produced the same pattern of error types, which led the latter to conclude: "The effect of providing feedback for correct selections seems to be to raise the accuracy while leaving the pattern of errors and the latency effects intact" (p. 275). This implicates the forward error as the primary error type in this task. Thus an explanation of forward errors would be a major part of any explanation of stimulus stringing. In an attempt to remove some of the ambiguities resulting from comparisons across two studies, the present study was conducted to determine the effect of feedback for correct selections in the specific task used by Richardson and Warzak (1981). 


\section{METHOD}

\section{Subjects}

Seven racing homing pigeons, deprived to $75 \%$ of their freefeeding weights, served as subjects. They had had over 170 sessions of training (see Richardson \& Warzak, 1981, for initial training conditions) on the color-string task used in the present experiment.

\section{Apparatus}

The two test chambers had inside dimensions of $53 \mathrm{~cm}$ long, $35 \mathrm{~cm}$ wide, and $37 \mathrm{~cm}$ high. A 75-dB (re $20 \mathrm{SPL}$ ) white noise was continuously present, and a fan provided ventilation.

$A$ jeweled houselight was centered on one wall, $3 \mathrm{~cm}$ from the ceiling. Opposite to it was a test panel with two rows of five circular openings, $2.5 \mathrm{~cm}$ in diameter. The lower row was $22 \mathrm{~cm}$ above the floor, and the upper row was $7.5 \mathrm{~cm}$ (center to center) above the lower row. The lower row (keys) served to present stimuli and to record responses. The upper row (stimulus windows) served to present one of the two types of experimenter-programmed feedback for correct selections. A Scientific Prototype food cup on the test-panel wall was illuminated when food $(45-\mathrm{mg}$ Noyes pigeon pellet) was delivered.

Industrial Electronics Engineers Series 10 inline-display cells containing four Wratten filters (Nos. 65, 74, 99, 73) with peak hue transmission values of $501,538,555$, and $576 \mathrm{~nm}$ were located behind the keys. Each stimulus could be off, dimly illuminated $(5.0 \mathrm{~V})$, or brightly illuminated $(6.3 \mathrm{~V})$. Transparent Lexan paddles between the opening and the display cell were operated by a static force of $5-15 \mathrm{~g}(.05-.15 \mathrm{~N})$ through an excursion of $.5-1.0 \mathrm{~mm}$

An Interdata 732 computer controlled the experimental task and data collection.

\section{Procedure}

A set of 10 arrays (arrangements of the colors on the keys) was chosen so that color and key were not confounded (see Richardson \& Warzak, 1981, Table 1, array-block 2, for the specific arrays used) in randomized blocks of 20 trials; each array occurred twice in each block.

The correct order of colors was a continuous spectral sequence of 501, 538, 555, and $576 \mathrm{~nm}$ for Group A (Subjects 201, 203, 205, and 207) and a mixed spectral sequence of 555, 501, 576, and $538 \mathrm{~nm}$ for Group B (Subjects 202, 206, and 208).

Each session was preceded by a 5 -min adaptation period in the dark chamber. At the beginning of each trial in the initial (baseline) condition, four dimly lighted colors (A, B, C, and D) and an unlighted surface were presented in random positions on the keys. Each correct selection produced two kinds of feedback: the pecked key changed from dim to bright (on-key feedback), and the same color sppeared in the leftmost unoccupied stimulus window (off-key feedback). After correct strings, the food-cup light was lighted for $2 \mathrm{sec}$ and, if scheduled, food was delivered. An incorrect selection resulted in offset of all displays and a .1-sec buzzer activation. A discrete-trials procedure with a 5-sec dark-key intertrial interval and between-trials correction was used so that each array was repeated until a correct pecking sequence had occurred. Each session was terminated after 100 trials or $60 \mathrm{~min}$, whichever came first. After the last trial, all lights went off. Food was delivered for correct strings on a variable-ratio 3 schedule. The subjects were given only 10 sessions of training under the baseline condition since they had had considerable training under this condition in previous experiments.

In the second condition, which was in effect for 26 sessions, the on-key feedback for correct selections was removed. In the third condition, which lasted for 20 sessions, both the on-key feedback and the off-key feedback for correct selections were removed; thus, in this condition there was no experimenter-programmed feedback for correct selections. During the final condition $(8$ sessions), the buzzer feedback for incorrect selections was also removed. Conditions were changed when there was no systematic trend in accuracy.
It appeared that one peck would occasionally operate the response switch twice very rapidly, so interresponse times less than $150 \mathrm{msec}$ were not counted. Excluding pecks with very short interresponse times prevented these second switch operations from being counted as errors.

\section{RESULTS}

Accuracy of performance (number of correct strings/ total number of strings) is plotted in Figure 1 for blocks of two sessions. The data from the two groups have been combined, since an analysis of variance did not show a difference in accuracy for the last three sessions of baseline $[F(1,6)=.46]$ or for the last three sessions of the no on-key feedback condition $[F(1,6)=.03]$.

Repeated measures analysis of variance of accuracy was performed using the initial and final three sessions of some conditions. Accuracy dropped when the on-key feedback was removed $[F(1,6)=43.15$, $p<.001]$, and then increased by the end of the no on-key feedback condition $[\mathrm{F}(1,6)=9.86, \mathrm{p}=.02]$. However, accuracy at the end of the no on-key feedback condition was still below baseline $[F(1,6)=9.86$, $\mathrm{p}<.0251$. Accuracy remained stable thereafter, as shown by Figure 1 and the comparison of the end of the no on-key feedback condition with the initial $[F(1,6)=2.92]$ and final $[F(1,6)=.44]$ sessions of the no on-key and no off-key feedback condition. Also, the sessions effect $[F(2,12)=1.54]$ and the sessions $X$ conditions interaction $[F(2,12)=.54]$ were not statistically significant for the latter comparison. Finally, as accuracy dropped 8 points during the first session of the no on-key or off-key feedback condition, this change was analyzed and found not to reach significance at the .05 level $[F(1,6)=5.82]$. In summary, accuracy fell when the on-key feedback was removed,

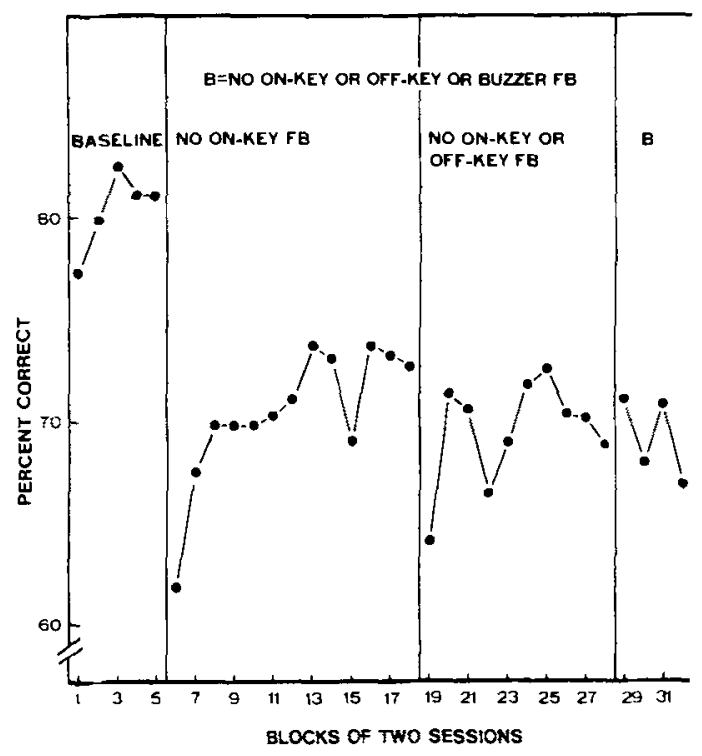

Figure 1. Accuracy as a function of blocks of two sessions. 
recovered some with training, but remained below baseline over the other conditions.

A more molecular analysis may be performed by grouping errors into four types. Errors resulting from a jump forward in the required sequence (e.g., A C) are called forward errors, while those resulting from a jump backward in the required sequence (e.g., A B C A) are called backward errors. Pecking the same stimulus twice in succession (e.g., A B B) is a repeat error, and pecks to the unlighted key during a trial are called dark-key errors. The different error types have different numbers of opportunities to occur, depending on the behavior of the subject, so the number of errors was converted to a relative frequency by dividing the number of errors of each type by the number of opportunities for that error type.

The number of opportunities was based on the number of selections (correct and incorrect) at each serial position of the sequence. As there was one unlighted key in each array, there was always one opportunity for a dark-key error at each serial position. Additionally, there were three opportunities for a forward error at Serial Position 1 (i.e., B, C, and D). In Serial Position 2 there were two opportunities for a forward error $(C, D)$ and one opportunity for a repeat error (A). In Serial Position 3, there was one opportunity for a forward error (D), one opportunity for a backward error (A), and one opportunity for a repeat error (B). Finally, in Serial Position 4, there were two opportunities for a backward error $(A, B)$ and one opportunity for a repeat error (C). The actual number of opportunities was empirically derived from each data set for each subject.

Error-type probabilities were analyzed for the last three sessions of baseline, the first three sessions of no on-key feedback, and the last three sessions of no on-key or off-key feedback. The probability of a dark-key error was zero or near zero for all subjects except Subject 203, which had probabilities of .0016, .0040 , and .0163 for the three conditions, respectively. Therefore, dark-key errors are not considered in the following analyses.

Table 1 presents the probability of each of the remaining error types for these three conditions. Analyses of variance showed a significant change over conditions for backward errors $[F(2,12)=6.21, p=$ $.014]$ and repeat errors $[F(2,12)=12.93, p=.001]$,

Table 1

Error Type Probability for Three Sessions Means at the End of Baseline, the Beginning of the No On-Key Feedback Condition, and the End of the No On-Key or Off-Key Feedback Condition

\begin{tabular}{lccc}
$\begin{array}{c}\text { Error } \\
\text { Type }\end{array}$ & Baseline & $\begin{array}{c}\text { No On-Key } \\
\text { Feedback }\end{array}$ & $\begin{array}{c}\text { No On-Key or } \\
\text { Off-Key Feedback }\end{array}$ \\
\hline Forward & .0259 & .0275 & .0355 \\
Backward & .0053 & .0197 & .0086 \\
Repeat & .0109 & .0726 & .0347 \\
\hline
\end{tabular}

but not forward errors $[F(2,12)=2.42]$. Post hoc comparisons (Newman-Keuls) showed the same pattern of change for both backward and repeat errors when the .05 level of significance was used. Specifically, the probabilities increased when the on-key feedback was removed, and then dropped by the end of the no on-key or off-key feedback condition, but remained above the baseline level.

Comparison of the three error types showed that forward errors had the highest probability under the baseline condition $[F(1,6)=8.95, p=.024$, for forward vs. repeat errors]. The increase in the probability of backward and repeat errors at the beginning of the no on-key feedback condition resulted in repeat errors' being the most probable for that condition $[F(1,6)=8.54, p=.027$, for forward vs. repeat errors] and forward and backward errors' not being different $[F(1,6)=3.77]$. By the end of the no on-key or off-key feedback condition, forward and repeat errors did not differ in probability $[F(1,6)=.01]$, but forward errors were more probable than backward errors $[F(1,6)=33.77, p=.001]$. The percent of total errors was $58.8 \%$ forward errors and $31.6 \%$ repeat errors under the no on-key or off-key feedback condition $[F(1,6)=11.56, p=.015]$. Note that the percentof-total-errors statistic showed a difference between forward and backward errors, while the probability measure showed no difference.

\section{DISCUSSION}

Richardson and Warzak (1981) compared two studies from different laboratories and concluded that feedback for correct selections in stimulus strings resulted in an increase in accuracy with little differential effect on the probability of different error types. A direct comparison of the feedback and no-feedback conditions in the present study showed that feedback did not affect forward or dark-key error probability but did reduce the probability of both backward and repeat errors. As the feedback could not serve as an $S^{\Lambda}$ for responses which would produce forward and dark-key errors, it was not surprising that they were unaffected. Backward and repeat errors, on the other hand, were affected, showing that the feedback did serve as an $S^{\Delta}$ for responses that would produce backward and repeat errors. That is, the probability of backward and repeat errors was reduced due to a bright/dim discrimination. In the no on-key or offkey feedback condition, the probability of backward errors was well below that for forward errors. This shows that, although the bright/dim discrimination did increase the difference in the probability of forward and backward errors, the difference still existed in the absence of a bright/dim discrimination. Explanation of forward errors is of major importance for understanding stimulus stringing. In the no-feedback condition, repeat errors were just as probable as for- 
ward errors. We have argued elsewhere (Richardson \& Warzak, 1981) that most repeat errors are probably due to the nature of the peck response for the pigeon and that they have more relevance for understanding pigeons than for understanding serial learning in general.

Straub et al. (1979) analyzed error types using the percent-of-total-errors statistic, a measure which does not take into account the number of opportunities to make each type of error. We have stressed the use of a probability measure as the dependent variable when examining error types. In the present study, the two measures were in clear disagreement for the comparison of forward and repeat errors in the no on-key or off-key feedback condition, in which the probability of these error types $(.0355$ vs. .0347) did not differ, but there was a higher proportion of forward errors $(58.8 \%$ vs. $31.6 \%)$. We believe the percent-of-total-errors measure to be misleading and inappropriate for the study of the effects of controlling variables on error types.

The similarity of behavior under the feedback and no-feedback conditions indicates that the underlying process is the same for both conditions and that the primary effect of feedback for pigeon subjects is to suppress the probability of repeat errors. If repeat pecks were not counted as errors, as was the case in the Straub et al. (1979) study, adding feedback should have little effect on the stringing behavior. The implication is that stimuli which lead to backward errors are strongly discriminated in some way from those leading to forward errors in the absence of any differential external stimulus conditions. As there is no lasting external stimulus change which would allow discrimination of the two categories of responses, the difference must rely on internal stimulus conditions. The underlying process may be short-term memory (Roberts, 1972); that is, the basic variable controlling the relative magnitude of forward and backward errors may be time since the last response to stimuli which, if selected, would generate backward errors. The birds learn not to peck stimuli which have recently been pecked. Increased feedback for correct responses reduces backward errors by transferring control, at least in part, from short-term memory to the feedback stimulus which is a permanent part of the environment. Transient feedback for correct selections (c.g., a light flash) should decrease the probability of backward errors relative to the no-feedback condition by effecting a more intense internal stimulus.

One explanation of forward errors (Straub et al., 1979 ) is that the subject pecks at a stimulus without closing the key and then pecks the next stimulus in the sequence, generating a forward error. The present data are in disagreement with this hypothesis (the inadequate-peck hypothesis). When feedback was given, adequate pecks were clearly indicated by the resultant feedback. The feedback was shown to control behavior by reducing the probability of the subjects' responding to the same stimulus twice in succession or going backward in the sequence. If inadequate pecks occurred, the feedback should reduce the probability of going to the next stimulus in the sequence after inadequate pecks. Instead, the subject should peck the inadequately pecked stimulus again until the feedback is produced. The results of the present study showed that the feedback variable did not affect the probability of forward errors, thus reducing the credibility of the inadequate-peck hypothesis.

\section{REFERENCES}

Richardson, W. K., \& Warzak, W. J. Stimulus stringing by pigeons. Journal of the Experimental Analysis of Behavior, $1981,36,267-276$.

Roberts, W. A. Short-term memory in the pigeon: Effects of repetition and spacing. Journal of Experimental Psychology, $1972,94,74-83$.

Straub, R. O., Seidenberg, M. S., Bever, T. G., \& Terrace, H. S. Serial learning in the pigeon. Journal of the Experimental Analysis of Behavior, 1979, 32, 137-148.

(Manuscript received June 29, 1981; revision accepted for publication October 12, 1981.) 